\title{
Theory of attosecond delays in molecular photoionization
}

\section{Journal Article}

\section{Author(s):}

Baykusheva, Denitsa; Wörner, Hans Jakob

Publication date:

2017-03-28

Permanent link:

https://doi.org/10.3929/ethz-a-010890568

Rights / license:

In Copyright - Non-Commercial Use Permitted

Originally published in:

The Journal of Chemical Physics 146(12), https://doi.org/10.1063/1.4977933

Funding acknowledgement:

307270 - Measuring attosecond electron dynamics in molecules (EC) 


\title{
Theory of attosecond delays in molecular photoionization
}

\author{
Denitsa Baykusheva and Hans Jakob Wörner* \\ Laboratorium für Physikalische Chemie, ETH Zürich, \\ Vladimir-Prelog-Weg 2, 8093 Zürich, Switzerland
}

(Dated: October 28, 2016)

\begin{abstract}
We present a theoretical formalism for the calculation of attosecond delays in molecular photoionization. It is shown how delays relevant to one-photon ionization, also known as Eisenbud-WignerSmith delays, can be obtained from the complex dipole-matrix elements provided by molecular quantum scattering theory. These results are used to derive formulae for the delays measured by two-photon attosecond interferometry based on an attosecond pulse train and a dressing femtosecond infrared pulse. These effective delays are first expressed in the molecular frame where maximal information about the molecular photoionization dynamics is available. The effects of averaging over the emission direction of the electron and the molecular orientation are introduced analytically. We illustrate this general formalism for the case of two polyatomic molecules. $\mathrm{N}_{2} \mathrm{O}$ serves as an example of a polar linear molecule characterized by complex photoionization dynamics resulting from the presence of molecular shape resonances. $\mathrm{H}_{2} \mathrm{O}$ illustrates the case of a non-linear molecule with comparably simple photoionization dynamics resulting from a flat continuum. Our theory establishes the foundation for interpreting measurements of the photoionization dynamics of all molecules by attosecond metrology.
\end{abstract}

*woerner@phys.chem.ethz.ch; www.atto.ethz.ch 


\section{INTRODUCTION}

In recent years, the techniques of attosecond spectroscopy have led to observation and control of electron dynamics in atoms, molecules and solids. One important branch of attosecond spectroscopy was initiated by the study of the real-time dynamics of photoionization, starting with photoemission from solids [1-3] and single-photon ionization of atoms $[4,5]$. These measurements have explored the natural attosecond time scale on which pho-

toelectrons leave the parent species [6-9] with a recent emphasis on the role of atomic resonances $[10,11]$. In the case of atoms, the accepted interpretation of these measurements relies on the Eisenbud-Wigner-Smith time delay that can be defined for most scattering processes and more or less universal delays caused by the measurement [12-15]. Photoemission from solids is a much more complicated process in which the atomic contributions to the delay should also be important $[2,16,17]$. The field of photoemission delays has been comprehensively reviewed in Ref. [18].

Attosecond photoionization delays from molecules have received surprisingly little attention so far, presumably because of the associated experimental and theoretical complexity. Very recently, we have reported measurements of photoionization delays of $\mathrm{N}_{2} \mathrm{O}$ and $\mathrm{H}_{2} \mathrm{O}$ molecules $[28,29]$ together with a brief summary of a theory that is fully outlined and developed in this article. Previously reported theoretical approaches to time-resolved molecular photoionization comprise calculations based on the solution of the full time-dependent Schrödinger equation for relatively simple prototypical systems such as $\mathrm{H}_{2}^{+}$([19-24]), or restricted one-dimensional single-active-electron models of diatomic molecules ([25, 26]). Delays for one-photon ionization of $\mathrm{N}_{2}$ and $\mathrm{CO}$ calculated using a Schwinger variational procedure were reported in [27]. In Section II of this article, we argue that the definition of the photoionization delays given in Ref. [27] is incorrect. More importantly, we show that there is no simple additive relation between one-photon-ionization delays of molecules and those measured by attosecond interferometry. This difference between atoms and molecules arises from the fact that (i) molecules lack spherical symmetry such that multiple partial waves are required in the description of the initial bound state and (ii) the photoionization matrix elements depend on the orientation of the molecule relative to the ionizing radiation, such that delays measured in partially to randomly aligned molecules differ from those measured in the molecular frame in a non-trivial manner. Despite its inherent complexity, the 
understanding of molecular photoionization delays will however offer an attractive bridge between the complex world of the condensed phase and the transparent case of atoms. It also constitutes an essential step in extending attosecond metrology to molecular forms of matter, comprising clusters, liquids and solids.

We now discuss the novelty of the information that can be obtained from attosecond timeresolved measurements of photoionization delays, as compared to more traditional variants of photoelectron spectroscopy. A complete description of photoionization within the dipole approximation requires the knowledge of the amplitude and phase of the transition dipole matrix elements from the bound initial state to the final continuum states. Photoelectron spectroscopy is frequently used to measure energy- and final-state resolved partial cross sections. These cross sections can be expressed as a sum of squared magnitudes of partial-wave matrix elements and are therefore not sensitive to the phase of these matrix elements. Photoelectron angular distributions, in contrast, are defined by interference between different partial waves and are therefore sensitive to the phase shifts between degenerate continua [30]. However, time-independent measurements can only determine phase differences between photoelectron continua belonging to the same ionization threshold. Phase relations between ionization continua corresponding to different ionization energies cannot, in principle, be measured because the corresponding photoelectrons have a different kinetic energy. The techniques of time-domain spectroscopy do enable such measurements, provided that the bandwidth covered by the ionizing radiation exceeds the energetic separation of the considered ionization thresholds. Measurements of photoionization delays in molecules do therefore provide qualitatively new information on the dynamics of photoionization which is not accessible to frequency-domain methods. For this reason, comparisons of such measurements with theory are particularly interesting because they test a previously unexplored aspect of scattering calculations.

This article is structured as follows. Section II defines time delays in molecular photoionization. Starting from the (Eisenbud-Wigner-Smith) delays associated with single-photon ionization, defined in the molecular frame, we show how the delays measured by attosecond interferometry are obtained, first in the molecular frame and then in the laboratory frame. Section III illustrates these results for the case of two recently experimentally investigated molecules $\mathrm{N}_{2} \mathrm{O}$ and $\mathrm{H}_{2} \mathrm{O}$ [29] which illustrate the complementary cases of linear and non-linear molecules, and additionally reveal the role of shape resonances on molecular 
photoionization delays. Section IV contains the conclusions of this article.

\section{TIME DELAYS IN MOLECULAR PHOTOIONIZATION}

The concept of time delay was introduced by Wigner [31] and Eisenbud [32] from a timedependent perspective in the context of the scattering of wavepackets composed of spherical waves. By defining the concept of a "dwell time", Smith [33] arrived at an expression connecting the time delay with the properties of stationary eigenstates, in particular the S-matrix. While these concepts were restricted initially to the special case of short-range potential scattering, the concept of time-delay was shown after appropriate modifications to be transferable to systems subject to Coulomb interaction [34, 35], a situation relevant for the case of photoionization. In the next section, we apply this concept to the case of molecular photoionization and outline the calculation of molecular time delays form state-of-the-art molecular quantum scattering theory currently employed in the theories of photoionization and photoelectron spectroscopies. The treatment of molecular targets involves several conceptual difficulties that have to be accommodated in the time-delay formalism introduced by Smith. These include, in addition to the presence of multiple channels, the angular dependence introduced by the directional dependence of the photoelectron on the one hand and the orientation of the molecule with respect to the laboratory-frame axes on the other hand. We first consider the case of single-photon ionization, whereby we first employ the "angular time delay" concept as introduced by Froissart, Goldberger and Watson [36] to treat the angular dependence of molecular photoionization and discuss the procedure for averaging over the photoemission angle and the molecular orientation. Considering the fact that current experimental schemes targeting photoionization time delays involve the interaction with a second laser pulse, displaced in time relative to the ionizing extreme-ultraviolet (XUV) radiation, we next consider the case of two-photon ionization by one high-frequency (typically XUV) photon and one low-frequency (typically infrared, IR) photon. This case is relevant for experiments relying on interferometry with attosecond pulse trains (APT), also used in the reconstruction of attosecond beating by interference of two-photon transitions (RABBIT) scheme [37]. The effects of spatial averaging over the photoelectron emission angle and the molecular orientation are also discussed. 


\section{A. One-photon-ionization delays}

Throughout this text, we use the method of single-center partial-wave expansion around the center of mass to study the photoionization of molecular targets. The actual scattering calculation is performed numerically using ePolyScat [38, 39], which is based on the Schwinger variational principle [40]. The formalism employed in the current section closely follows the notation employed in [41]. For brevity, the notation is restricted to the singlechannel case, but generalization to the multi-channel case is straightforward. As discussed in [42], the scattering operator in terms of which Smith's time delay definition is constructed, can be related to the photoionization matrix element. The photoionization dipole matrix elements expressed in the length gauge are of the general form:

$$
I_{i, f}=\left\langle\Psi_{i}|\vec{r} \cdot \hat{n}| \Psi_{f, \vec{k}}^{(-)}\right\rangle,
$$

where $\left|\Psi_{i}\right\rangle$ is the initial state, $\vec{r}$ is the position operator and $\hat{n}$ denotes the polarization (propagation) direction for linearly (circularly) polarized light. Here and in what follows, atomic units are used, unless otherwise stated. The final (residual ion + photoelectron) wavefunction is expanded into partial waves:

$$
\Psi_{f, \vec{k}}^{(-)}=\sqrt{\frac{2}{\pi}} \sum_{l m} i^{l} \Psi_{f, l m}{ }^{(-)}(\vec{r}) Y_{l m}^{*}(\hat{k}),
$$

where $\hat{k} \equiv\left(\theta_{k}, \phi_{k}\right)$ denotes the emission direction of the photoelectron with the asymptotic momentum $k$ in the molecular frame of reference. The partial-wave states $\Psi_{f, l m}^{(-)}(\vec{r})$ are solutions of the Lippmann-Schwinger equation defined in [40]. The dipole moment operator

$\vec{r} \cdot \hat{n}$ can be written explicitly in spherical tensor form $\hat{\xi}_{m_{\mathrm{p}}} \equiv \vec{r} \cdot \hat{n}=r Y_{1 m_{\mathrm{p}}}\left(\hat{r}^{\prime}\right)$ where $m_{\mathrm{p}}=0$ for linearly and \pm 1 for circularly polarized light and $\hat{r}^{\prime}$ denotes the position operator defined with respect to the laboratory frame (LF). In our treatment, the photon polarization (propagation direction) is chosen to define the $Z$-axis of the LF in the case of linear (circular) polarization. The relation between this frame and the molecule-fixed frame (MF) employed in eq. (1) can be expressed in terms of the Euler angles $\left(\hat{R}_{\gamma} \equiv(\alpha, \beta, \gamma)\right)$ defined in fig. 1 that rotate the laboratory frame into coincidence with the molecular frame. In the molecular frame, the dipole-transition operator reads:

$$
\hat{\xi}_{m_{\mathrm{p}}}=\sqrt{\frac{4 \pi}{3}}\left(1-2 \delta_{1 m_{\mathrm{p}}}\right) r \sum_{\mu=-1}^{1} \mathcal{D}_{\mu m_{\mathrm{p}}}^{(1)}\left(\hat{R}_{\gamma}\right) Y_{1 \mu}(\hat{r})
$$


where $\mathcal{D}_{m k}^{(j)}\left(\hat{R}_{\gamma}\right)$ denotes the Wigner matrix element. Therefore one can expand the dipole moment $I_{i, f}$ defined in (1) as:

$$
I_{i, f}=\sum_{l m \mu} I_{l m \mu} Y_{l m}(\hat{k}) \mathcal{D}_{\mu m_{\mathrm{p}}}^{(1)}\left(\hat{R}_{\gamma}\right)
$$

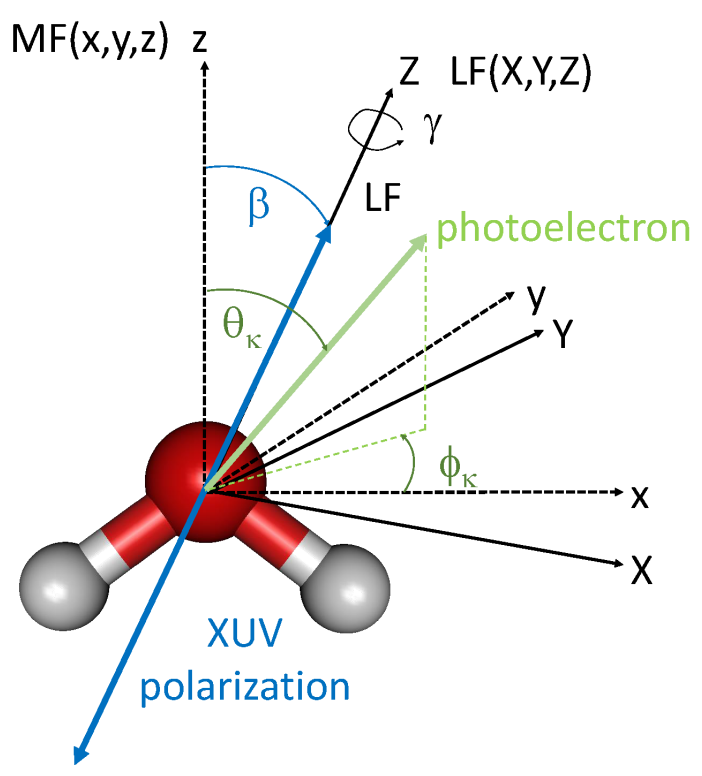

FIG. 1. Definition of the coordinate systems (molecular frame, MF, and laboratory frame, LF), the set of Euler angles $\hat{R}_{\gamma}=(\alpha, \beta, \gamma)$ transforming the MF into the LF and the angles $\left(\theta_{\kappa}, \phi_{\kappa}\right)$ defining photoemission.

Following the reasoning of Froissart, Goldberger and Watson [36], we can define the "angular time delay" in the context of molecular photoionization as:

$$
\tau_{1 h \nu}\left(E, \hat{k}, \hat{R}_{\gamma}\right)=\hbar \frac{\partial}{\partial E} \arg \left\{I_{i, f}\right\}=\hbar \frac{\partial}{\partial E} \arg \left\{\sum_{l m \mu} I_{l m \mu} Y_{l m}^{*}(\hat{k}) \mathcal{D}_{\mu m_{\mathrm{p}}}^{(1)}\left(\hat{R}_{\gamma}\right)\right\} .
$$

Equation (5) defines a delay associated with single-photon ionization for photoemission along the direction $\hat{k}$ in the molecular frame for a particular orientation $\left(\hat{R}_{\gamma}\right)$ of the molecule with respect to the ionizing radiation. In the following, we refer to such quantities as emission-angle (or short angle-) and target-orientation (or short orientation-) resolved delays. We note that Eq. (5) differs from the definition given in Eq. (4) of Ref. [27], which we consider to be incorrect. Equation (5) indeed represents the energy derivative of the complex photoionization amplitude, which represents the group delay of the outgoing photoelectron wave packet, in line with the historical work in Refs. [32, 43-45] and the recent review 
article [18]. In contrast, Eq. (4) of Ref. [27] associates the photoionization delays with the derivative of the wave function of the continuum electron, which is not an adequate definition.

The formula given in eq. (5) cannot be directly applied to describe an experimental situation sampling the time delay only as a function of one of the solid angles $\hat{k}$ or $\hat{R}_{\gamma}$ while the resolution with respect to the other is absent. The correct procedure for performing

spatial averaging over $\tau\left(E, \hat{k}, \hat{R}_{\gamma}\right)$ must take into account the contribution of a particular emission direction to the total photoionization cross section. The method to achieve this consists in weighting the delay associated with a particular set of angles $\hat{k}$ and $\hat{R}_{\gamma}$ with the corresponding cross section:

$$
\tau_{1 h \nu}(E)=\hbar \int \mathrm{d} \hat{R}_{\gamma} \int \mathrm{d} \hat{k} \frac{\left|\sum_{l m \mu} I_{l m \mu} Y_{l m}^{*}(\hat{k}) \mathcal{D}_{\mu m_{\mathrm{p}}}^{(1)}\left(\hat{R}_{\gamma}\right)\right|^{2}}{\sum_{l m \mu}\left|I_{l m \mu}\right|^{2}} \frac{\partial}{\partial E} \arg \left\{\sum_{l m \mu} I_{l m \mu} Y_{l m}^{*}(\hat{k}) \mathcal{D}_{\mu m_{\mathrm{p}}}^{(1)}\left(\hat{R}_{\gamma}\right)\right\} .
$$

This expression defines the one-photon-ionization delay averaged over both emission angles and target orientation, i.e. the result of a non-angle-resolved measurement on a randomlyoriented molecular sample. A formula similar to expression (6) for the case of scattering on a short-range potential has been derived and discussed by Nussenzveig [45] [46].

\section{B. Two-photon delays in molecular photoionization}

\section{Angle- and orientation-resolved delays}

In the following we derive the two-photon matrix elements describing photoionization delays measured by a combination of XUV and IR laser pulses, typical of attosecond interferometry. In doing so, we make the following assumptions. First, the XUV photon energy $\Omega$ is assumed to be much larger than the relevant ionization potential $\left(I_{\mathrm{p}}\right)$ of the molecule. This allows one to ignore bound-state contributions to the two-photon matrix elements. Second, ionization pathways in which the XUV photon is absorbed after the IR photon of energy $\omega$ are neglected. The two-photon ionization matrix element for a fixed-in-space target in the molecular frame (MF) is then given by:

$$
M\left(\vec{k} ; \varepsilon_{i}+\Omega\right)=\frac{1}{\mathrm{i}} E_{\mathrm{XUV}} E_{\mathrm{IR}} \lim _{\epsilon \rightarrow 0^{+}} \int \frac{\left\langle\Psi_{f, \vec{k}}^{(-)}(\vec{r})\left|\hat{\xi}_{m_{\mathrm{p}}^{\prime}}^{\mathrm{IR}}\right| \nu\right\rangle\left\langle\nu\left|\hat{\xi}_{m_{\mathrm{p}}}^{\mathrm{XUV}}\right| \Psi_{i}\right\rangle}{\varepsilon_{i}+\Omega-\varepsilon_{\nu}+\mathrm{i} \epsilon},
$$


where $\Psi_{f, \vec{k}^{(-)}}^{(\vec{r})}$ and $\Psi_{i}$ are the final and initial states defined in the preceding section, while $|\nu\rangle$ denotes an intermediate continuum state with energy $\varepsilon_{\nu}$. We choose a Hartree-Fock wave function description for the initial state $|i\rangle$, as implemented in ePolyScat. Generalization to configuration-interaction wave functions is straightforward. We now perform a singlecenter partial-wave decomposition of the initial-state $\Psi_{i}=\sum_{l m} R_{n, l} Y_{l m}(\hat{r})$ and intermediatestate wave functions $|\nu\rangle=\sum_{\lambda \mu} R_{\nu \lambda}(r) Y_{\lambda \mu}(\hat{r})$. Since we restrict our analysis to the case where the energy of the XUV pulse exceeds the ionization potential of the system, the integral over the intermediate states labelled $\nu$ in eq. (7) effectively involves only partialwave components of the accessible intermediate continuum states. In analogy to eq. (2), $R_{\kappa \lambda}$ is also chosen such that the incoming-wave boundary conditions are satisfied. We treat the final-state wavefunction $\Psi_{f, \vec{k}^{(-)}}(\vec{r})$ within the frozen-core Hartree-Fock approximation, assuming a single-determinant representation of the final state in which the ionic orbitals are identical to the ones used for the description of the ground state. The photoelectron orbital corresponds to the solution of the one-electron Schrödinger equation with a potential defined by the static-exchange interaction with the molecular ion, a short-range potential term and the Coulomb interaction [47]. Generalization to configuration-interaction wave functions and inclusion of orbital relaxation is conceptually straightforward.

We separate the final state given by eq. (2) explicitly in terms of radial and angulardependent parts:

$$
\Psi_{f, \vec{k}}^{(-)}(\vec{r})=\sqrt{\frac{2}{\pi}} \sum_{L M} \mathrm{i}^{L} \mathrm{e}^{-\mathrm{i} \eta_{L}(\hat{k})} Y_{L M}^{*}(\hat{k}) Y_{L M}(\hat{r}) R_{k L}(r),
$$

where the radial solution $R_{k L}$, corresponding to continuum momentum $k$, satisfies the proper incoming-wave boundary conditions, $\hat{k}$ captures the angular dependence of the ejected electron and $\eta_{L}(\hat{k})=\delta_{L}(\hat{k})+\sigma_{L}(\hat{k})$ is the partial-wave phase shift that consists of a short-range $\left(\delta_{L}(\hat{k})\right)$ and Coulomb $\left(\sigma_{L}(\hat{k})\right)$ part. The general expression in eq. (7) defines the two-photonionization amplitude for a fixed-in-space target in the molecular frame. Thus, the transition operators $\hat{\xi}^{\mathrm{XUV} / \mathrm{IR}}$ are tied to the MF. The polarizations of the XUV/IR fields, however, are defined with respect to the LF and correspondingly, $\hat{\xi}^{\mathrm{XUV}} / \hat{\xi}^{\mathrm{IR}}$ have to be transformed to the MF according to eq. (3). From now on, the dependence of $M\left(\vec{k} ; \varepsilon_{i}+\Omega\right)$ on the target orientation will be denoted explicitly: $M\left(\vec{k} ; \varepsilon_{i}+\Omega\right) \rightarrow M\left(\vec{k} ; \varepsilon_{i}+\Omega ; \hat{R}_{\gamma}\right)$. In addition, we 
adopt the following convention for the indices for the polarizations of the XUV/IR fields:

$$
\begin{gathered}
\rho^{\prime}, m_{1} \rightarrow \mathrm{XUV} \\
\rho, m_{2} \rightarrow \mathrm{IR} .
\end{gathered}
$$

After inserting all partial-wave expansions into (7) and evaluating the angle-dependent integrals, we obtain:

$$
\begin{aligned}
M\left(\vec{k} ; \varepsilon_{i}+\Omega ; \hat{R}_{\gamma}\right)= & \frac{1}{\mathrm{i}} \frac{1}{16 \pi^{2}} E_{\mathrm{XUV}} E_{\mathrm{IR}} \sqrt{\frac{2}{\pi}}\left(1-2 \delta_{1 m_{1}}\right)\left(1-2 \delta_{1 m_{2}}\right) \\
& \sum_{\substack{L M \\
\rho \rho^{\prime} \\
l m}}(-\mathrm{i})^{L} \mathrm{e}^{\mathrm{i} \eta_{L}(k)}(-1)^{\rho+\rho^{\prime}} \sqrt{2 l+1} \sqrt{2 L+1}(2 \lambda+1) \\
& \left\langle 1 \rho^{\prime} \lambda \mu \mid l m\right\rangle\langle 10 \lambda 0 \mid l 0\rangle\langle 1-\rho L M \mid \lambda \mu\rangle\langle 10 L 0 \mid \lambda 0\rangle \\
& T_{L \lambda l}\left(k ; \varepsilon_{\kappa}\right) \mathcal{D}_{\rho^{\prime} m_{1}}^{(1)}\left(\hat{R}_{\gamma}\right) \mathcal{D}_{\rho m_{2}}^{(1)}\left(\hat{R}_{\gamma}\right) Y_{L M}(\hat{k}),
\end{aligned}
$$

where $\langle\ldots \mid \ldots\rangle$ denotes the Clebsch-Gordan coefficient and $T_{L \lambda l}\left(k ; \varepsilon_{\kappa}\right)$ is the radial part of the two-photon transition matrix element:

$$
T_{L \lambda l}\left(k ; \varepsilon_{\kappa}\right)=\sum_{\nu, \varepsilon_{\nu}<0} \frac{\left\langle R_{k L}|r| R_{\nu \lambda}\right\rangle\left\langle R_{\nu \lambda}|r| R_{n l}\right\rangle}{\varepsilon_{i}+\Omega-\varepsilon_{\nu}}+\lim _{\epsilon \rightarrow 0^{+}} \int_{0}^{\infty} \mathrm{d} \varepsilon_{\kappa^{\prime}} \frac{\left\langle R_{k L}|r| R_{\kappa^{\prime} \lambda}\right\rangle\left\langle R_{\kappa^{\prime} \lambda}|r| R_{n l}\right\rangle}{\varepsilon_{i}+\Omega-\varepsilon_{\kappa^{\prime}}+\mathrm{i} \epsilon} .
$$

As in [14], $T_{L \lambda l}\left(k ; \varepsilon_{\kappa}\right)$ can be evaluated using the Dalgarno-Lewis approach. In the asymptotic region, we can write $T_{L \lambda l}\left(k ; \varepsilon_{\kappa}\right)$ as:

$$
\begin{aligned}
T_{L \lambda l}\left(k ; \varepsilon_{\kappa}\right) \approx & \frac{1}{|\kappa-k|^{2}} \exp \left[-\frac{\pi Z}{2}\left(\frac{1}{\kappa}-\frac{1}{k}\right)\right] \mathrm{i}^{L-\lambda-1} \mathrm{e}^{\mathrm{i}\left(\eta_{\lambda}(\hat{\kappa})-\eta_{L}(\hat{k})\right)} \\
& \frac{(2 \kappa)^{\mathrm{i} Z / \kappa}}{(2 k)^{\mathrm{i} Z / k}} \frac{\Gamma\left[2+\mathrm{i} Z\left(\kappa^{-1}-k^{-1}\right)\right]}{(\kappa-k)^{(1 / \kappa-1 / k)}}\left\langle R_{\kappa \lambda}|r| R_{n l}\right\rangle .
\end{aligned}
$$

Setting

$$
A_{\kappa k}=\frac{1}{|\kappa-k|^{2}} \exp \left[-\frac{\pi Z}{2}\left(\frac{1}{\kappa}-\frac{1}{k}\right)\right] \frac{(2 \kappa)^{\mathrm{i} Z / \kappa}}{(2 k)^{\mathrm{i} Z / k}} \frac{\Gamma\left[2+\mathrm{i} Z\left(\kappa^{-1}-k^{-1}\right)\right]}{(\kappa-k)^{1 / \kappa-1 / k}}
$$

results in:

$$
\begin{aligned}
M\left(\vec{k} ; \varepsilon_{i}+\Omega ; \hat{R}_{\gamma}\right) & =\frac{1}{16 \pi^{2}} E_{\mathrm{XUV}} E_{\mathrm{IR}} \sqrt{\frac{2}{\pi}}\left(1-2 \delta_{1 m_{1}}\right)\left(1-2 \delta_{1 m_{2}}\right) A_{\kappa k} \\
& \sum_{\substack{L M \\
\rho \rho^{\prime} \\
l m}}(-\mathrm{i})^{\lambda} \mathrm{e}^{\mathrm{i} \eta_{\lambda}(\kappa)}(-1)^{\rho+\rho^{\prime}} \sqrt{2 l+1} \sqrt{2 L+1}(2 \lambda+1) \\
& \left\langle 1 \rho^{\prime} \lambda \mu \mid l m\right\rangle\langle 10 \lambda 0 \mid l 0\rangle\langle 1-\rho L M \mid \lambda \mu\rangle\langle 10 L 0 \mid \lambda 0\rangle \\
& \left\langle R_{\kappa \lambda}|r| R_{n l}\right\rangle \mathcal{D}_{\rho^{\prime} m_{1}}^{(1)}\left(\hat{R}_{\gamma}\right) \mathcal{D}_{\rho m_{2}}^{(1)}\left(\hat{R}_{\gamma}\right) Y_{L M}(\hat{k}) .
\end{aligned}
$$


The quantity $A_{\kappa k}$ can be identified as an IR-induced, "continuum-continuum" part of the two-photon amplitude as it does not depend on the target structure within the asymptotic approximation used here. One can also identify the photoionization matrix element corresponding solely to the XUV-absorption step:

$$
I_{\lambda \mu \rho^{\prime}}:=\sqrt{\frac{2}{\pi}}(-\mathrm{i})^{\lambda} \mathrm{e}^{\mathrm{i} \eta_{\lambda}(\kappa)}(2 \lambda+1)(-1)^{\rho^{\prime}} \sum_{l m} \sqrt{2 l+1}\left\langle 1 \rho^{\prime} \lambda \mu \mid l m\right\rangle\langle 0 \lambda 0 \mid l 0\rangle\left\langle R_{\kappa \lambda}|r| R_{n l}\right\rangle .
$$

We note that eq. (16) corresponds to the complex-conjugate of the PI-matrix element defined in [41]. With these auxiliary definitions, the matrix element

$$
\begin{aligned}
M\left(\vec{k} ; \varepsilon_{i}+\Omega ; \hat{R}_{\gamma}\right)= & \frac{1}{16 \pi^{2}} E_{\mathrm{XUV}} E_{\mathrm{IR}} A_{\kappa k}\left(1-2 \delta_{1 m_{1}}\right)\left(1-2 \delta_{1 m_{2}}\right) \\
& \sum_{\substack{L M \\
\rho \rho^{\prime} \\
\lambda \mu}} \sqrt{2 L+1}(-1)^{\rho}\langle 1-\rho L M \mid \lambda \mu\rangle\langle 10 L 0 \mid \lambda 0\rangle \\
& I_{\lambda \mu \rho^{\prime}} \mathcal{D}_{\rho^{\prime} m_{1}}^{(1)}\left(\hat{R}_{\gamma}\right) \mathcal{D}_{\rho m_{2}}^{(1)}\left(\hat{R}_{\gamma}\right) Y_{L M}(\hat{k})
\end{aligned}
$$

can be cast in a concise form:

$$
M\left(\vec{k} ; \varepsilon_{i}+\Omega ; \hat{R}_{\gamma}\right)=\frac{1}{16 \pi^{2}} E_{\mathrm{XUV}} E_{\mathrm{IR}} A_{\kappa k} \sum_{L M} b_{L M}^{m_{1}, m_{2}}\left(\hat{R}_{\gamma}\right) Y_{L M}(\hat{k})
$$

where the orientation-dependent coefficients $b_{L M}^{m_{1}, m_{2}}\left(\hat{R}_{\gamma}\right)$ are defined as:

$$
\begin{aligned}
b_{L M}^{m_{1}, m_{2}}\left(\hat{R}_{\gamma}\right)= & \sum_{\rho \rho^{\prime}} b_{L M}^{m_{1}, m_{2} ; \rho \rho^{\prime}} \mathcal{D}_{\rho^{\prime} m_{1}}^{(1)}\left(\hat{R}_{\gamma}\right) \mathcal{D}_{\rho m_{2}}^{(1)}\left(\hat{R}_{\gamma}\right) \\
= & \left(1-2 \delta_{1 m_{1}}\right)\left(1-2 \delta_{1 m_{2}}\right) \sum_{\substack{\rho \rho^{\prime} \\
\lambda \mu}} \sqrt{2 L+1}(-1)^{\rho} \\
& \langle 1-\rho L M \mid \lambda \mu\rangle\langle 10 L 0 \mid \lambda 0\rangle I_{\lambda \mu \rho^{\prime}} \mathcal{D}_{\rho^{\prime} m_{1}}^{(1)}\left(\hat{R}_{\gamma}\right) \mathcal{D}_{\rho m_{2}}^{(1)}\left(\hat{R}_{\gamma}\right) .
\end{aligned}
$$

In the following, we discuss the two-photon delays accessible in a RABBIT measurement. Up to this point, our equations are valid for both linearly and circularly polarized radiation and arbitrary relative polarizations of XUV and IR. For simplicity, we now restrict the polarizations of XUV and IR fields to be linear and parallel to each other, i.e. $m_{1}=m_{2}=0$ and the superscripts in the $b_{L M}^{m_{1}, m_{2}}$-coefficients will be omitted for brevity. The angle-resolved intensity of a photoelectron sideband corresponding to energy $2 q \omega(q \in \mathbb{N})$ created in an attosecond interferometry experiment is given by:

$$
\begin{aligned}
\frac{\mathrm{d}^{2} P_{2 q}}{\mathrm{~d} \hat{k} \mathrm{~d} \hat{R}_{\gamma}} & \propto\left|M^{(2 q-1)}+M^{(2 q+1)}\right|^{2}=\left|M^{(2 q-1)}\right|^{2}+\left|M^{(2 q+1)}\right|^{2} \\
& +2\left|M^{(2 q-1)}\right|\left|M^{(2 q+1)}\right| \cos \left[\arg \left\{M^{(2 q-1) *} M^{(2 q+1)}\right\}\right],
\end{aligned}
$$


where $\frac{k^{2}}{2}=2 q \omega-I_{\mathrm{p}}$ and $\frac{\kappa^{2}}{2}=(2 q \pm 1) \omega-I_{\mathrm{p}}$.

The angle- and orientation-resolved delay in the finite-difference approximation [14] reads:

$$
\tau\left(2 q, \hat{k}, \hat{R}_{\gamma}\right)=\frac{1}{2 \omega} \arg \left(M^{(2 q-1) *} M^{(2 q+1)}\right)
$$

where

$$
M^{(2 q-1) *} M^{(2 q+1)}=A_{\kappa k}^{(2 q-1) *} A_{\kappa k}^{(2 q+1)} \sum_{\substack{L M \\ L^{\prime} M^{\prime}}} b_{L^{\prime} M^{\prime} ;(2 q-1)}^{*}\left(\hat{R}_{\gamma}\right) b_{L M ;(2 q+1)}\left(\hat{R}_{\gamma}\right) Y_{L^{\prime} M^{\prime}}^{*}(\hat{k}) Y_{L M}(\hat{k}) .
$$

This quantity contains the maximal available information about the attosecond photoionization dynamics and can be written as a sum of two terms:

$$
\tau\left(2 q, \hat{k}, \hat{R}_{\gamma}\right)=\tau_{\text {cc }}(2 q)+\tau_{\text {mol }}\left(2 q, \hat{k}, \hat{R}_{\gamma}\right)
$$

The structure of eq. (23) shows that measurements of molecular photoionization delays in the MF by attosecond interferometry, just as their atomic counterparts, can be interpreted in terms of a continuum-continuum contribution (or measurement-induced delay) [5, 14],

$$
\tau_{\mathrm{cc}}(2 q)=\frac{1}{2 \omega} \arg \left[A_{\kappa k}^{(2 q-1) *} A_{\kappa k}^{(2 q+1)}\right]
$$

which only depends on the involved photon energies. However, the angular momentum addition describing the interaction with the IR photon leads to a non-trivial modification of the angle dependence of the two-photon-ionization delays compated to their one-photon counterpart that we factorize into a molecule-specific contribution [29]:

$$
\tau_{\text {mol }}\left(2 q, \hat{k}, \hat{R}_{\gamma}\right)=\frac{1}{2 \omega} \arg \left[\sum_{\substack{L M \\ L^{\prime} M^{\prime}}} Y_{L^{\prime} M^{\prime}}^{*}(\hat{k}) Y_{L M}(\hat{k}) b_{L^{\prime} M^{\prime} ;(2 q-1)}^{*}\left(\hat{R}_{\gamma}\right) b_{L M ;(2 q+1)}\left(\hat{R}_{\gamma}\right)\right] .
$$

Although molecular photoionization delays can, in principle, be measured with angular resolution for fixed-in-space molecules, such experiments have not been reported to date. We therefore now subsequently discuss the effect of angular and target-orientation averaging.

\section{Effect of averaging over emission angle and target orientation}

In the following, we describe the application of these results to several possible configurations of experimental interest. We first introduce the effect of averaging over the emission 
angle $\hat{k}$ of the photoelectron. A product of the form of eq. (22) is easily uniformly averaged over $\hat{k}$ by exploiting the orthonormality of the spherical harmonics, leading to:

$$
\tau\left(2 q, \hat{R}_{\gamma}\right)=\tau_{\mathrm{cc}}(2 q)+\frac{1}{2 \omega} \arg \left[\sum_{L M} b_{L M ;(2 q-1)}^{*}\left(\hat{R}_{\gamma}\right) b_{L M ;(2 q+1)}\left(\hat{R}_{\gamma}\right)\right] .
$$

This quantity represents the delay measured from perfectly oriented molecules by averaging over the emission angle of the photoelectron.

Measurements of molecular photoionization delays have so far only been reported for unaligned samples [29]. This situation is described by additionally averaging over all molecular orientations, leading to

$$
\tau(2 q)=\tau_{\text {cc }}(2 q)+\frac{1}{2 \omega} \arg \left[\sum_{L M} \int \mathrm{d} \hat{R}_{\gamma} b_{L M ;(2 q-1)}^{*}\left(\hat{R}_{\gamma}\right) b_{L M ;(2 q+1)}\left(\hat{R}_{\gamma}\right)\right] .
$$

Molecular photoionization delays could also be measured by aligning and orienting molecules in space using non-resonant laser pulses [48-51] or by post-selection in coincidence/covariance experiments for cases where dissociative photoionization pathways can be cleanly separated and the axial-recoil approximation is valid. In all such cases, averaging must be performed over a characteristic axis distribution $A\left(\hat{R}_{\gamma}\right)$ and the corresponding delays are given by

$$
\tau(2 q)=\tau_{\text {cc }}(2 q)+\frac{1}{2 \omega} \arg \left[\sum_{L M} \int \mathrm{d} \hat{R}_{\gamma} A\left(\hat{R}_{\gamma}\right) b_{L M ;(2 q-1)}^{*}\left(\hat{R}_{\gamma}\right) b_{L M ;(2 q+1)}\left(\hat{R}_{\gamma}\right)\right]
$$

Finally, a measurement of angle-resolved delays could be performed for a randomly aligned molecular sample. This situation is described by uniformly averaging over $\hat{R}_{\gamma}$ only, but not over $\hat{k}$ :

$$
\tau(2 q, \hat{k})=\tau_{\mathrm{cc}}(2 q)+\frac{1}{2 \omega} \arg \left[\sum_{\substack{L M \\ L^{\prime} M^{\prime}}} Y_{L^{\prime} M^{\prime}}^{*}(\hat{k}) Y_{L M}(\hat{k}) \int \mathrm{d} \hat{R}_{\gamma} b_{L^{\prime} M^{\prime} ;(2 q-1)}^{*}\left(\hat{R}_{\gamma}\right) b_{L M ;(2 q+1)}\left(\hat{R}_{\gamma}\right)\right] .
$$

\section{Comparison to the atomic case}

At this point, it is pertinent to outline the differences between the molecular case discussed so far and the result for an atomic system. Specifically, we compare Eq. (15) from this article with Eq. (24) of Ref. [14]. 
The first difference arises from the lack of spherical symmetry of molecules and applies both in the molecular and in the laboratory frames. The absence of spherical symmetry requires the summation over $\ell$, the angular-momentum quantum number of the partial waves involved in the expansion of the initial bound state. In contrast, the initial bound state of atoms can be described by a single value of $\ell$. Additionally, exploiting the Fano propensity rule [52], which states that the transition $\ell \rightarrow \ell+1$ strongly dominates over the transition $\ell \rightarrow \ell-1$, the phase of the two-photon matrix elements for atoms can be reduced to a sum containing the scattering phase $\eta_{\ell+1}(\kappa)$ and the cc-phase (the argument of Eq. (14) in this article), as done in Ref. [5]. Consequently, the two-photon delay accessible in angle-integrated RABBIT measurements of atoms can be written as

$$
\tau(2 q)=\tau_{\mathrm{cc}}(2 q)+\tau_{1 h \nu}(2 q)
$$

i.e. as a sum of a one-photon-ionization delay (the Wigner delay) and a continuumcontinuum delay. In the case of molecules, this separability does not exist in the same form, because the initial state cannot be represented by a single partial wave. Multiple partial waves are required to represent the initial state, therefore multiple partial waves are also required to represent the intermediate (one-photon-ionized) state, which leads to interference phenomena, e.g. between the pathways $\ell \rightarrow \ell+1$ and $\ell+2 \rightarrow \ell+1$ etc. Additional interferences occur at the level of the two-photon transitions, e.g. between $\ell \rightarrow \ell+1 \rightarrow \ell$ and $\ell+2 \rightarrow \ell+1 \rightarrow \ell$.

For these reasons, the two-photon delays in molecular photoionization cannot be written as a sum of a one-photon-ionization delay and a universal continuum-continuum contribution. In other words, there is no simple additive relation between $\tau\left(2 q, \hat{R}_{\gamma}\right)$ and $\tau_{1 h \nu}\left(2 q, \hat{R}_{\gamma}\right)$ in the molecular case. However, owing to the independence of $\tau_{\mathrm{cc}}$ on the values of the angularmomentum quantum numbers, one can still isolate a "continuum-continuum contribution" (see Eq. (23)), but the residual $\tau_{\text {mol }}(2 q)$ has no simple relation to $\tau_{1 h \nu}(2 q)$. Consequently, the effect of the probing IR field on the delays measured in the molecular case cannot be represented by a simple, universal quantity. Instead, one has to first evaluate Eq. (19), followed by Eqs. (25) to (29), depending on the case of experimental interest.

The second difference to the atomic case is the dependence of all matrix elements on the orientation of the target molecule, i.e. the dependence on the Euler angles $\hat{R}_{\gamma}$, which is explicitly given in the preceding equations. The dependence of the delays on $\hat{R}_{\gamma}$ is very pro- 
nounced, as we show below, and it plays a crucial role in averaging over the axis distribution, which is relevant for all experiments. The non-trivial aspect of the orientational-averaging arises from the fact that Eqs. (27) to (29) represent coherent integrals over complex quantities. This fact can completely change the energy-dependence of $\tau_{\text {mol }}(2 q)$, as we illustrate in Section III.C below. We show that the coherent averaging over molecular orientations can shift the position of the maximal delay caused by a shape resonance by $\sim 7 \mathrm{eV}$ when comparing the one-photon-ionization delays $\tau_{1 h \nu}$ with the molecular part of the two-photon delays $\tau_{\mathrm{mol}}$.

\section{APPLICATIONS}

We apply the expressions derived in the preceding section to the case of two polyatomic molecules that have recently been investigated experimentally using attosecond interferometry, i.e. $\mathrm{N}_{2} \mathrm{O}$ and $\mathrm{H}_{2} \mathrm{O}$ [29]. The initial-state single-determinant wavefunction $\Psi_{i}$ was obtained from a Hartree-Fock quantum chemistry calculation using the 3-21G basis set and bond lengths of $R(\mathrm{~N}-\mathrm{N})=1.207 \AA$ and $R(\mathrm{~N}-\mathrm{O})=1.237 \AA$ in the case of $\mathrm{N}_{2} \mathrm{O}$. In the case of $\mathrm{H}_{2} \mathrm{O}$, the cc-pVTZ basis set was used with bond lengths of $R(\mathrm{O}-\mathrm{H})=0.9578 \AA$ and a bond angle of $\theta(\mathrm{HOH})=104.5^{\circ}$. The choice of a rather small basis in the case of $\mathrm{N}_{2} \mathrm{O}$ is justified by the fact that the scattering calculations correctly reproduce the experimentally observed behaviour of the photoionization cross sections and the asymmetry parameters (cp. supplemental material of [29]), in particular the position of the shape resonances in the $7 \sigma(\mathrm{HOMO}-1) \rightarrow k \sigma$ and $7 \sigma \rightarrow k \pi$ channels, that we discuss below). In both cases, we employ the frozen nuclei approximation and perform the scattering calculation keeping fixed bond distances and angles.

\section{A. Delays in one-photon ionization}

We first illustrate the angle- and orientation-resolved delays in one-photon ionization, as defined by eq. (5). These delays for ionization from the $\tilde{\mathrm{X}}^{1} \Sigma^{+}$electronic ground state of $\mathrm{N}_{2} \mathrm{O}$ to the first electronically-excited state $\tilde{\mathrm{A}}^{+}{ }^{2} \Sigma^{+}$of $\mathrm{N}_{2} \mathrm{O}^{+}$are shown in fig. 2 for a molecule aligned parallel $((\alpha=0, \beta=0, \gamma=0)$, panel a $))$ or perpendicular $((\alpha=0, \beta=\pi / 2, \gamma=0)$, panel b)) to the polarization of the ionizing radiation. These choices of the Euler angles 

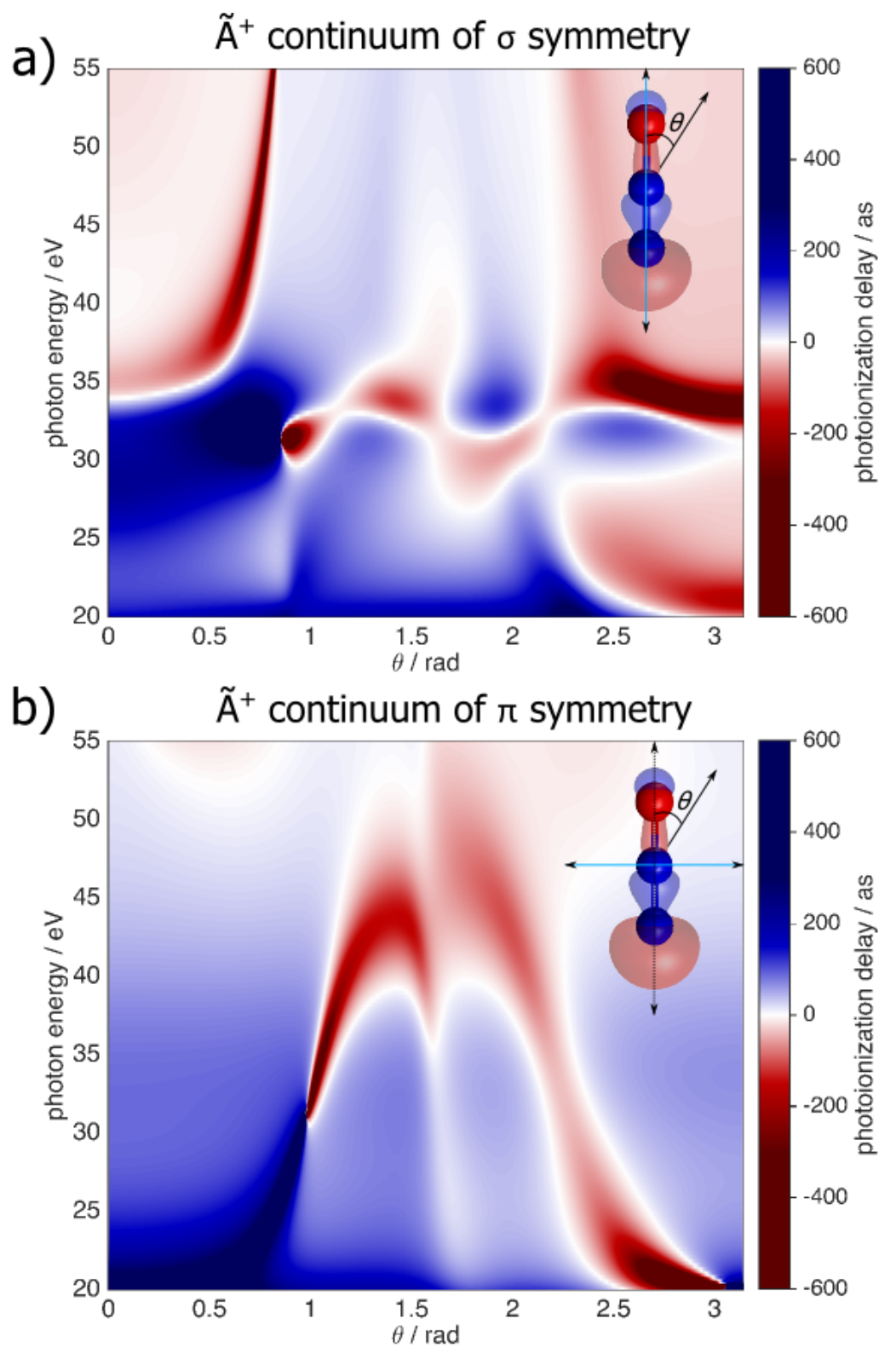

FIG. 2. Delays in the one-photon ionization of $\mathrm{N}_{2} \mathrm{O}$ to the $\tilde{\mathrm{A}}^{+}{ }^{2} \Sigma^{+}$state of $\mathrm{N}_{2} \mathrm{O}^{+}$, given in the molecular frame. a) XUV polarization parallel to the molecular axis, b) XUV polarization perpendicular to the molecular axis.

enable us to isolate the parallel transition to the $\sigma$ continuum from the perpendicular one, associated with the continuum of $\pi$-symmetry. The cylindrical symmetry of the $\mathrm{N}_{2} \mathrm{O}$ system lifts the dependence of the angle-resolved time delay on the azimuthal photoemission angle $\phi$. Figure 2 shows the delays as a function of the photoemission direction in the MF, quantified by the polar angle $\theta$ measured from the internuclear axis. 

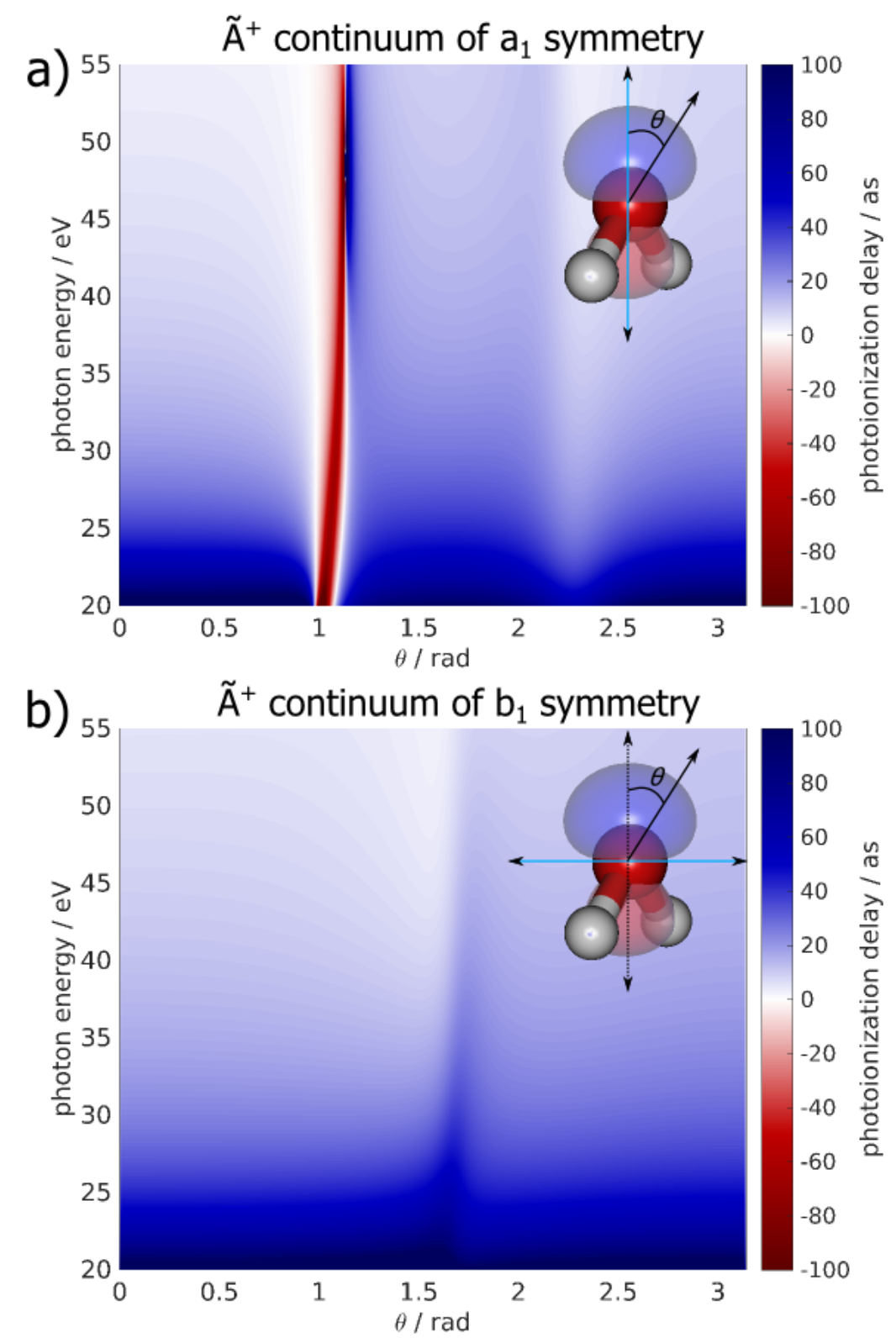

FIG. 3. Delays in the one-photon ionization of $\mathrm{H}_{2} \mathrm{O}$ to the $\tilde{\mathrm{A}}^{+}{ }^{2} \mathrm{~A}_{1}$ state of $\mathrm{H}_{2} \mathrm{O}^{+}$, given in the molecular frame. a) XUV polarization parallel to the principal axis, b) XUV polarization perpendicular to the principal axis.

The same quantities are illustrated in fig. 3 for the case of one-photon ionization from the $\tilde{\mathrm{X}}{ }^{1} \mathrm{~A}_{1}$ electronic ground state of $\mathrm{H}_{2} \mathrm{O}$ to the $\tilde{\mathrm{A}}^{+}{ }^{2} \mathrm{~A}_{1}$ electronically excited state of $\mathrm{H}_{2} \mathrm{O}^{+}$. The accessible continua have $a_{1}$ and $b_{1}$ symmetries in this case. The azimuthal angle $\phi$ has been set to 0 in this case. Note the use of different color scales in figs. 2 and 3 motivated by the fact that the delays are much smaller in magnitude in the case of $\mathrm{H}_{2} \mathrm{O}$ than in $\mathrm{N}_{2} \mathrm{O}$. 
At low photon energies, the angle-resolved delay maps of both $\mathrm{N}_{2} \mathrm{O}$ and $\mathrm{H}_{2} \mathrm{O}$ are dominated by large positive delays due to the attractive nature of the Coulomb potential, the regions around 2.5-3 rad in $\mathrm{N}_{2} \mathrm{O}$ and the narrow region centered around $1 \mathrm{rad}$ in $\mathrm{H}_{2} \mathrm{O}$ being exceptions to this trend. The most striking feature that becomes apparent when comparing these results is the richness of the angular structures in the angle-resolved photoionization time delays of $\mathrm{N}_{2} \mathrm{O}$ and the contrasting simplicity of the same quantities in $\mathrm{H}_{2} \mathrm{O}$.

The rich angular structure in the case of $\mathrm{N}_{2} \mathrm{O}$ is the signature of shape resonances embedded in the photoionization continua of this molecule. Previous work [53-60] in the realm of photoelectron spectroscopy has revealed a weak shape resonance associated with the $7 \sigma \rightarrow k \sigma$ channel in $\mathrm{N}_{2} \mathrm{O}$ located in the region around photon energies of $\sim 33 \mathrm{eV}[61]$. Notably, the single-photon angle-resolved time delay of the parallel transition in $\mathrm{N}_{2} \mathrm{O}$ (panel a) of fig. 2) is characterized by large values (in terms of absolute amplitude) and rapid variations as a function of the photoemission direction $\theta$ in this particular region $(30-35 \mathrm{eV})$. These delays are first strongly positive for small angles $(0.5-0.8 \mathrm{rad})$, then change rapidly in the region from 1 to $2.4 \mathrm{rad}$ and finally reach large negative values as evident from the "ridge" located at $\theta>2.5 \mathrm{rad}$. With increasing energies, the angular dependence of the delays becomes smoother, the sole exception being a narrow feature $(\theta \approx 0.5-0.6 \mathrm{rad})$ associated with strongly negative values of the delays. The transition to the $\pi$-continuum shown in fig. $2 \mathrm{~b}$ contains a very broad and weak shape resonance centered at around $38 \mathrm{eV}$ [61]. It is presumably the broadness of this resonance that leads to smearing out of the angular features in this region. Regions of large positive, abruptly followed by large negative delays are observed at $\theta \approx 1 \mathrm{rad}$ and around $35 \mathrm{eV}$, this feature becoming progressively less negative and moving towards higher angles with increasing energy.

In contrast to the non-uniform behaviour of the time delays in both channels of the $\tilde{\mathrm{A}}^{+}{ }^{2} \Sigma^{+}$-continuum of $\mathrm{N}_{2} \mathrm{O}$, the angular maps for the corresponding photoionization transitions in $\mathrm{H}_{2} \mathrm{O}$ are essentially monotonic. The predominantly negative delays and the relatively subtle angular dependencies (at least in Fig. 3b) reflect the dominant effect of the attractive Coulomb potential on the photoionization delays. The main exception to this trend is the narrow negative ridge centered at $\theta \approx 1 \mathrm{rad}$ extending through the entire energy range in Fig. 3a. This structure has an intuitive interpretation. Since the $\tilde{\mathrm{A}}^{+}{ }^{2} \mathrm{~A}_{1}$ state of $\mathrm{H}_{2} \mathrm{O}^{+}$ is well described by an electron being removed from the $3 \mathrm{a}_{1}$ orbital of dominant atomic p-character (see inset in Fig. 3a), the continuum is dominated by s- and d-waves. Since 
S-waves are spherically symmetric, whereas the d-waves accessible by symmetry change sign at the magic angle $\left(\theta \approx 54.7^{\circ} \approx 1.05 \mathrm{rad}\right)$, the total photoionization matrix elements to the continuum of $\mathrm{a}_{1}$ symmetry display a rapid variation of their amplitude and phase around 1 rad, which manifests itself as a local extremum in the photoionization delays. Photoionization by radiation polarized perpendicular to the $\mathrm{C}_{2}$ symmetry axis leads to the continuum of $b_{1}$ symmetry (Fig. 3b), dominated by d-waves of $d_{x z}$ symmetry (where $\mathrm{z}$ is the direction of the $\mathrm{C}_{2}$ axis and $\mathrm{x}$ the polarization direction of the ionizing radiation). Since we have restricted our analysis to the value $\phi=0$ for the emission direction, the contribution from the $b_{2}$ continuum accessible via a transition polarized along the molecule-fixed y-axis can be neglected as the dominant $\mathrm{d}_{\mathrm{yz}}$ waves have a nodal surface at this value of the azimuthal angle. The photoionization delays in the $b_{1}$ channel therefore display a rapid variation around $\theta \approx 90^{\circ} \approx \pi / 2 \mathrm{rad}$, where the matrix elements to the $\mathrm{d}_{\mathrm{xz}}$ continuum change sign, but not around $\theta \approx 1 \mathrm{rad}$, where the matrix elements vary smoothly with $\theta$ and energy, leading to very small angle-dependent delays.

Having outlined the relationship between the angle-resolved molecular delays and the structure of the corresponding continuum for one particular target orientation, we now turn to the effect of angular averaging over the photoemission and/or orientation directions. Figure 4 shows the delays for $\mathrm{N}_{2} \mathrm{O}$ (after coherently adding the contributions of the parallel and perpendicular transitions) as a function of the emission angle $\hat{k} \equiv \theta$ after averaging over the Euler angles (panel a)), or, alternatively, as a function of the Euler angle $\beta$ (the polar angle between the molecular and lab frames) after averaging over all emission directions (panel b)). In general, the integration with respect to either direction leads to a decrease in the absolute magnitude of the time delays, which motivates the use of a new color scale in fig. 4. The averaging over the direction of photoemission leads to an overall smearing and smoothing of the previously discussed features. However, the $7 \sigma \rightarrow k \sigma$-shape-resonance region remains identifiable as a region (around $33 \mathrm{eV}$ ) of locally increased photoionization delays for the entire range of $\beta$. Interestingly, the orientation-averaged, photoemission-angleresolved delays displayed in panel b) still feature a region with remarkably large positive values (localized at angles $\theta$ between 0.2 and $0.6 \mathrm{rad}$ and $2.4-2.8 \mathrm{rad}$ ) close to the above discussed shape resonance and extending towards the region near $40 \mathrm{eV}$. The comparison of Figs. 2 and 4 shows that the orientational averaging preserves a substantial amount of the time-delay structure observed in the molecular frame. However, the quantity represented in 

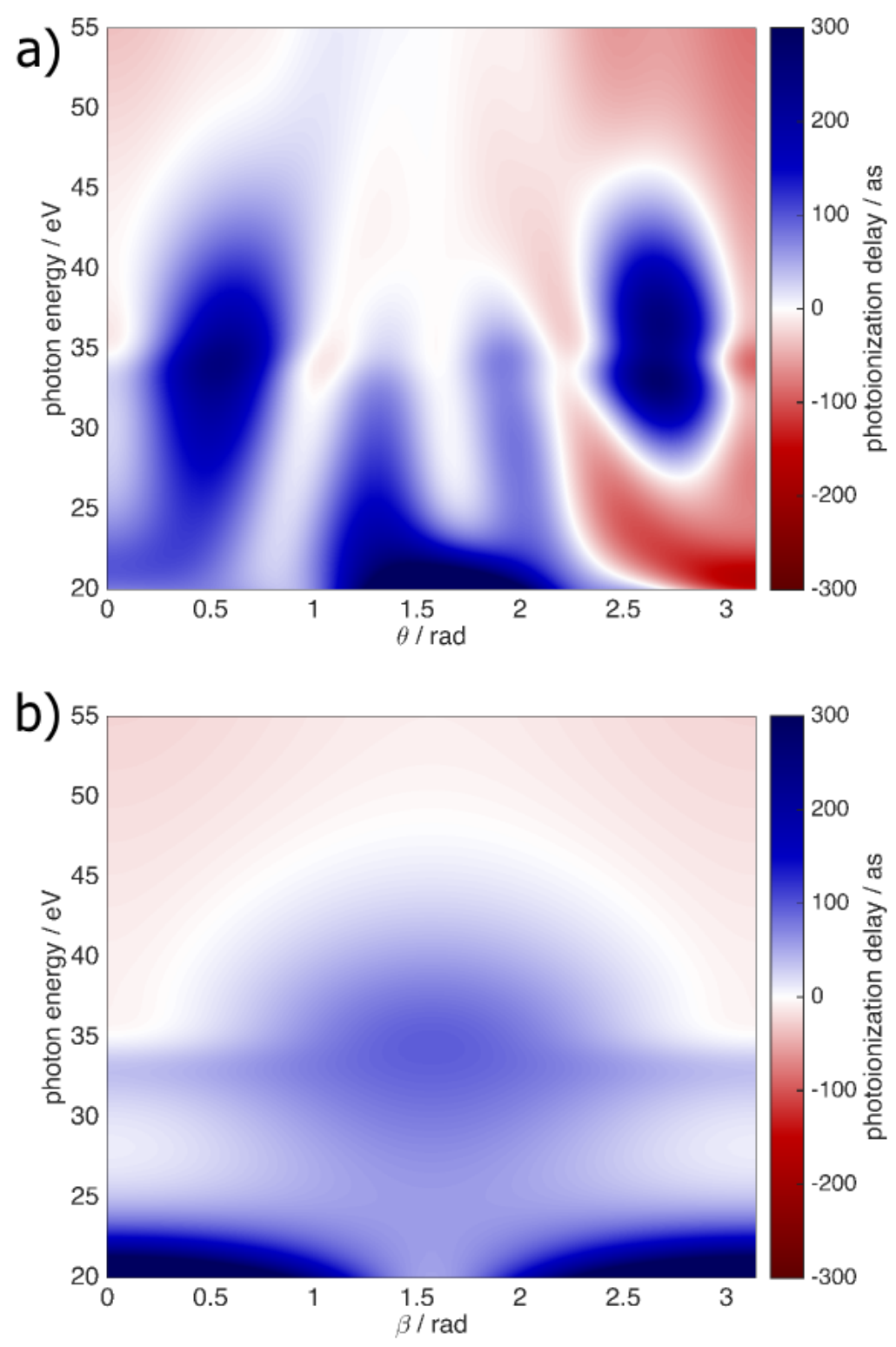

FIG. 4. a) Photoemission-angle-resolved one-photon photoionization delays of $\mathrm{N}_{2} \mathrm{O}$ to the $\mathrm{A}^{+}{ }^{2} \Sigma^{+}$state of $\mathrm{N}_{2} \mathrm{O}^{+}$, averaged over the molecular orientations according to eq. (6). b) Orientationresolved one-photon-ionization delays for the same system, averaged over the photoemission direction and reported as a function of the Euler angle $\beta$.

Fig. 4 is not related to the experimentally measured delays in a simple way, as discussed in Section II C. 


\section{B. Delays in two-photon ionization}

Attosecond interferometry relies on the use of synchronized XUV and IR pulses and consists in measuring the phase of the intensity oscillations of sidebands created by $1+1^{\prime}$ two-color photoionization. The effective photoionization delays obtained in such measurements are defined by eq. (21). These effective photoionization delays, just like the one-photon-ionization delays, have a non-trivial dependence on the angles $\hat{k}$ and $\hat{R}_{\gamma}$. Since these delays can however be written as a sum of an angle-independent term (eq. (24)) and an angle-dependent part $\left(\tau_{\text {mol }}\left(2 q, \hat{k}, \hat{R}_{\gamma}\right)\right.$, eq. $\left.(25)\right)$, we now illustrate the latter quantity for values of $\hat{R}_{\gamma}$ selected by symmetry. In the following, the IR photon energy is chosen to be $1.55 \mathrm{eV}$, corresponding to the most-frequently-used central wavelength of $800 \mathrm{~nm}$, and the XUV photon energy is treated as a continuous variable.

In analogy to the preceding section, we show in figs. 5 and 6 the emission-angle-resolved two-photon delays for the transitions $\tilde{\mathrm{X}}^{1} \Sigma^{+} \mathrm{N}_{2} \mathrm{O} \rightarrow \tilde{\mathrm{A}}^{+}{ }^{2} \Sigma^{+} \mathrm{N}_{2} \mathrm{O}^{+}$and $\tilde{\mathrm{X}}^{+}{ }^{1} \mathrm{~A}_{1} \mathrm{H}_{2} \mathrm{O} \rightarrow$ $\tilde{\mathrm{A}}^{+2} \mathrm{~A}_{1} \mathrm{H}_{2} \mathrm{O}^{+}$for $\beta=0$ and $\beta=\pi / 2$, respectively. The general features of the one-photon angle-dependent delay maps are preserved in the maps for the two-photon transitions. Both the sign and the magnitudes of the delays are comparable, but a detailed inspection of the

results reveals many differences. For example, a wide area of negative delays appears in Fig. 5a between $\theta=1.6$ and $2 \mathrm{rad}$ below energies of $\sim 29 \mathrm{eV}$, where the delays in Fig. 2a are positive. Similarly, a region of negative delays also appears between $\theta=0.8$ and $1 \mathrm{rad}$ between photon energies of 22 to $28 \mathrm{eV}$. Additionally, the angular variation of the one- and two-photon-ionization delays is also quite different, as illustrated by the comparison of the central regions of positive delay in Fig. 2a and 5a. Turning to Fig. 5b, we find more subtle changes as compared to Fig. 2b. However, a new region of positive delays appears below $\theta=0.2 \mathrm{rad}$, and the overall range of the delays is significantly reduced in Fig. 5b compared to Fig. 2b. A similar trend is also observed when comparing Figs. 3 and 6 . The main features are qualitatively very similar. However, the sharp ridge of negative delays for $\theta \approx$ $1 \mathrm{rad}$ in Fig. 3a is replaced by a line of weakly positive delays in Fig. 6a. The results shown in Figs. $3 \mathrm{~b}$ and $6 \mathrm{~b}$ are even more similar, except for the appearance of an additional ridge of small positive delays around $\theta \approx 0.2 \mathrm{rad}$ in Fig. $6 \mathrm{~b}$. 

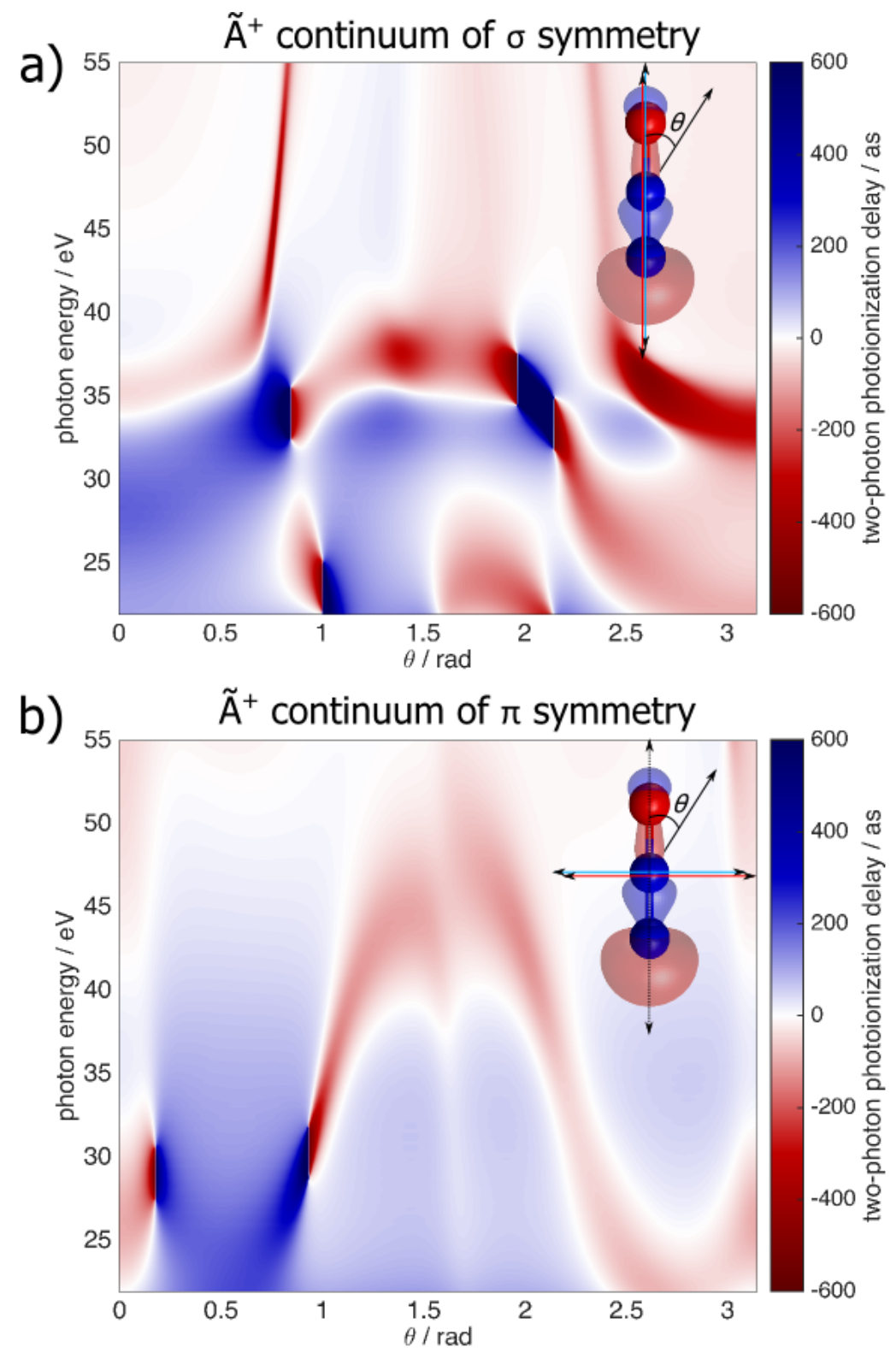

FIG. 5. Two-photon-ionization delays $\left(\tau_{\text {mol }}\right.$, as defined by eq. (25)) of $\mathrm{N}_{2} \mathrm{O}$ to the $\mathrm{A}^{+}{ }^{2} \Sigma^{+}$state of $\mathrm{N}_{2} \mathrm{O}^{+}$, given in the molecular frame. a) XUV and IR polarizations are both parallel to the molecular axis, b) XUV and IR polarizations are both perpendicular to the molecular axis. 

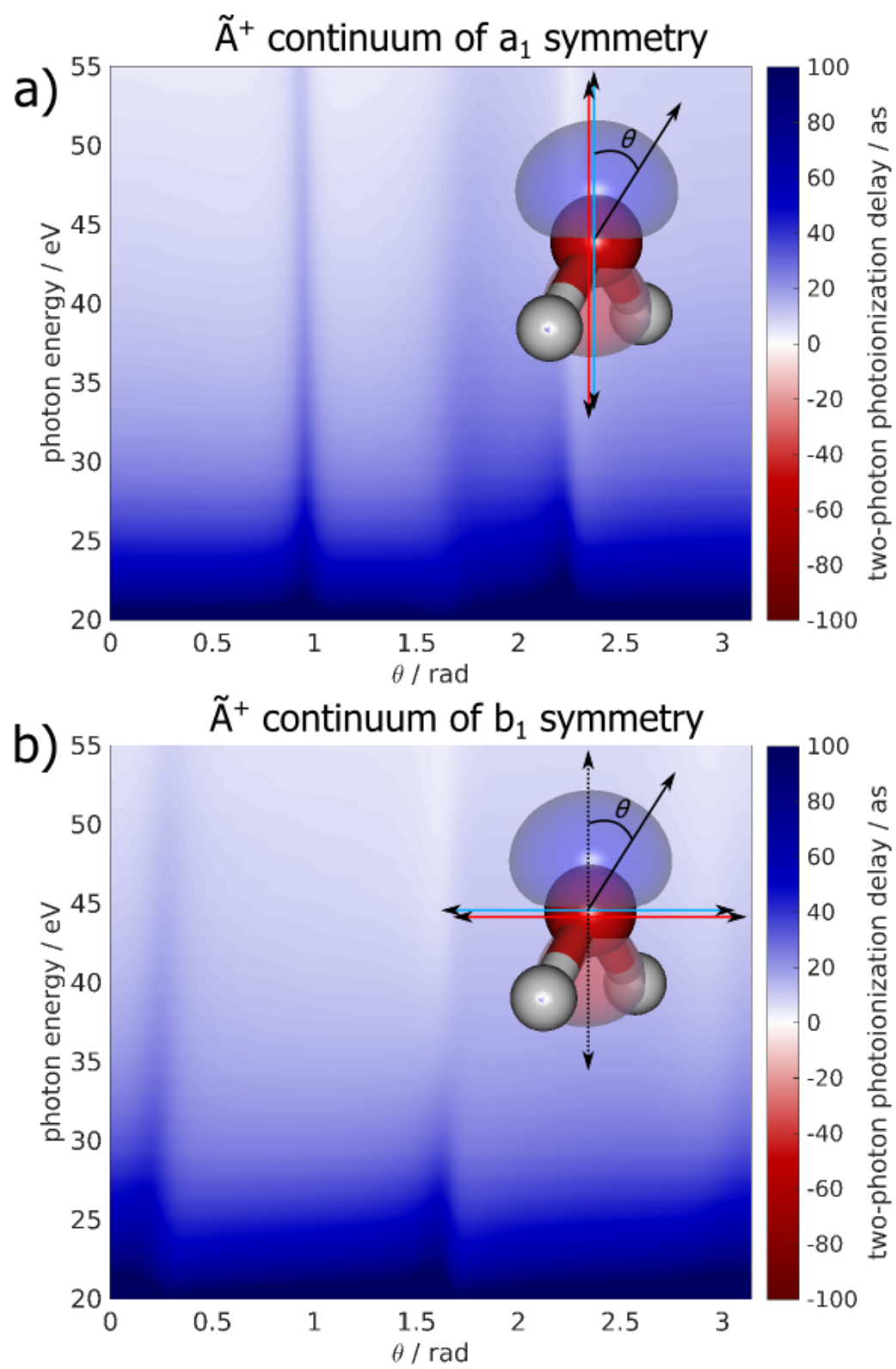

FIG. 6. Two-photon-ionization delays ( $\tau_{\text {mol }}$, as defined by eq. (25)) of $\mathrm{H}_{2} \mathrm{O}$ to the $\tilde{\mathrm{A}}^{+2} \mathrm{~A}_{1}$ state of $\mathrm{H}_{2} \mathrm{O}^{+}$, given in the molecular frame. a) XUV and IR polarizations are both parallel to the molecular axis, b) XUV and IR polarizations are both perpendicular to the molecular axis. 


\section{Comparison of angle-averaged delays in one- and two-photon ionization}

The preceding sections have illustrated the angle and orientation dependencies of the oneand two-photon-ionization delays. This comparison has shown that the angle-dependent features of the one-photon-ionization delays are qualitatively retained in the two-photonionization delays, but that the two types of delays differ on a quantitative level. This insight will be useful and important in future angle-resolved measurements of molecular photoionization delays. We now compare the effects of angular or orientational averaging on the one- and two-photon-ionization delays on a more quantitative level.
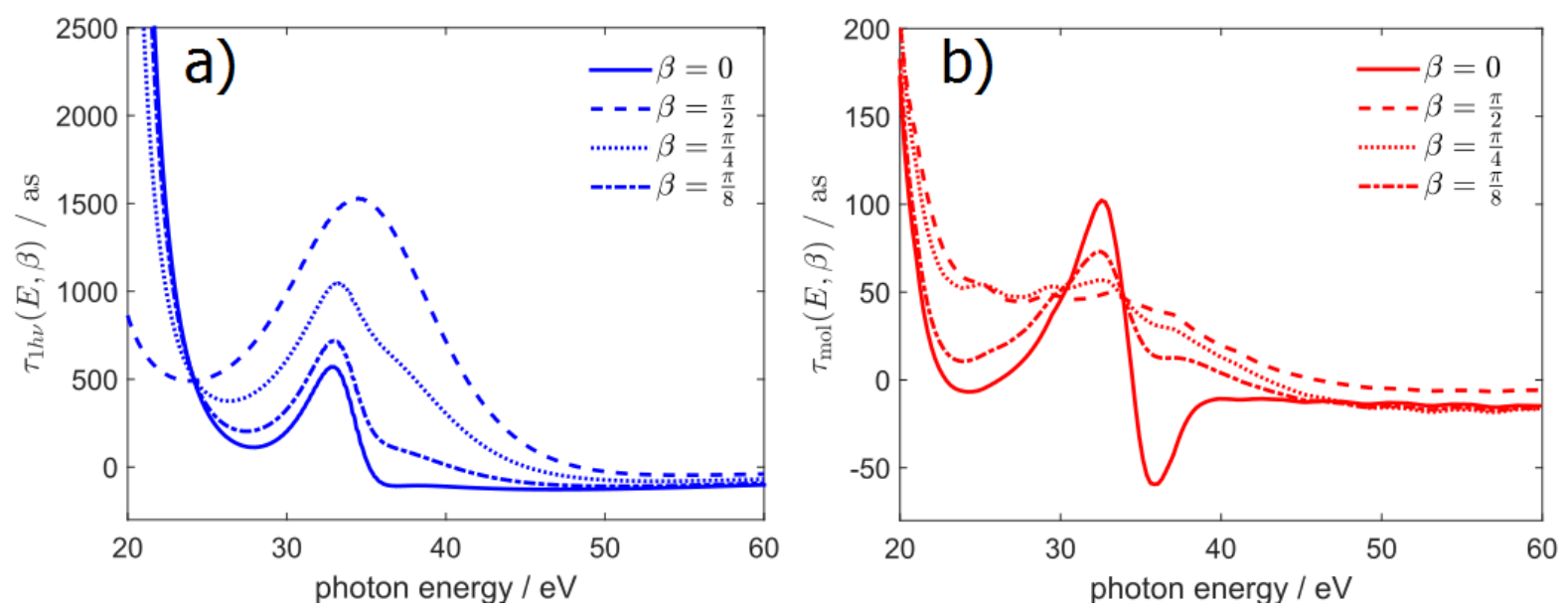

FIG. 7. Comparison of one- (a) and two-photon-ionization delays (b) for selected molecular orientations ( $\beta$ given in the legend) for the case of $\tilde{\mathrm{X}}^{1} \Sigma^{+} \mathrm{N}_{2} \mathrm{O} \rightarrow \tilde{\mathrm{A}}^{+}{ }^{2} \Sigma^{+} \mathrm{N}_{2} \mathrm{O}^{+}$after averaging over the photoemission direction.

We first compare in Fig. 7 the one- and two-photon-ionization delays for selected orientations ( $\beta$ values) of $\mathrm{N}_{2} \mathrm{O}$ after averaging over the direction of photoemission $(\theta)$. All delays display a local maximum which shifts from $32.8 \mathrm{eV}$ at $\beta=0 \mathrm{rad}$ to $34.5 \mathrm{eV}$ at $\beta=\pi / 2 \mathrm{rad}$ in panel (a) and from $32.5 \mathrm{eV}$ to $33.0 \mathrm{eV}$ in panel (b). The location of this maximum coincides well with the position of the shape resonance discussed above. Although the positions of the maxima are similar, we note that the magnitude of the delays and their energy dependencies differ substantially between the one- and two-photon-ionization delays. This is a consequence of the non-universal effect of the probe photon on molecular two-photon-ionization delays.

In a second step, we now investigate the even more significant impact of the additional averaging over the target orientation. Figure 8 shows the corresponding one- and two- 

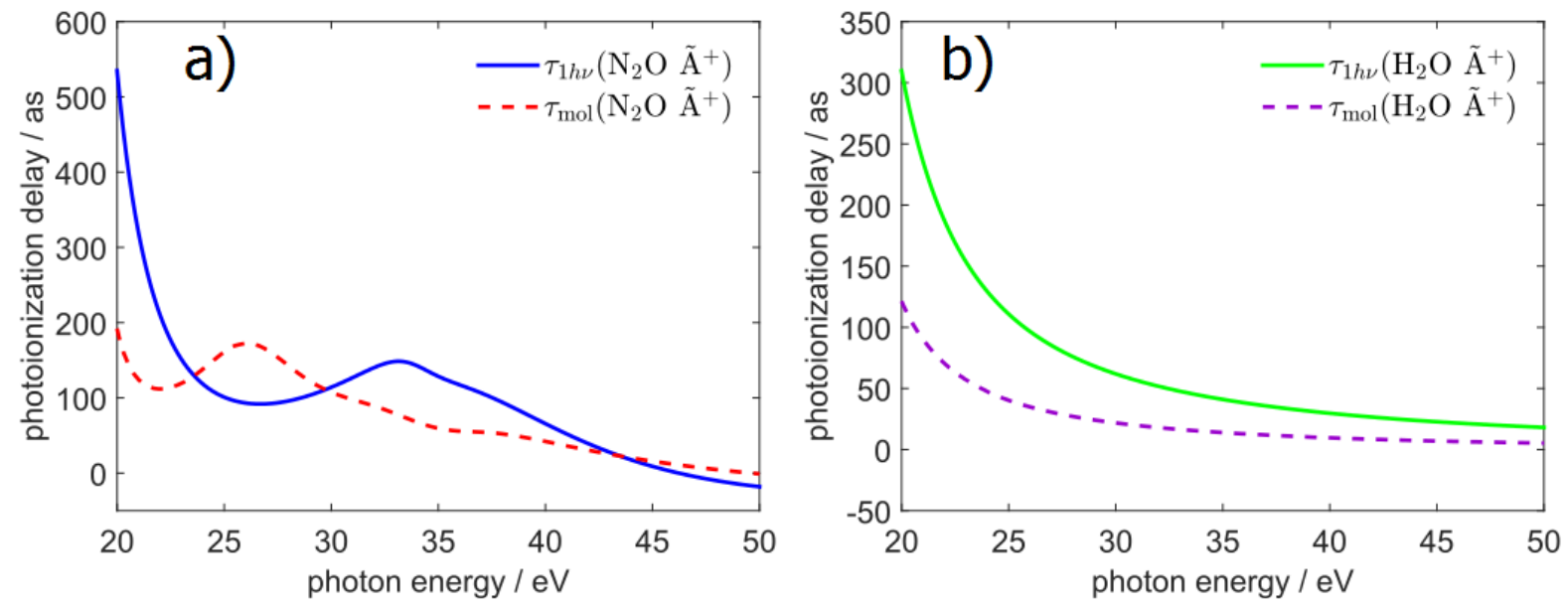

FIG. 8. Comparison of one- (solid lines) and two-photon-ionization (dashed lines) delays for the two investigated systems ( (a) $\tilde{\mathrm{X}}^{1} \Sigma^{+} \mathrm{N}_{2} \mathrm{O} \rightarrow \tilde{\mathrm{A}}^{+}{ }^{2} \Sigma^{+} \mathrm{N}_{2} \mathrm{O}^{+}$, and (b) $\tilde{\mathrm{X}}^{1} \mathrm{~A}_{1} \mathrm{H}_{2} \mathrm{O} \rightarrow \tilde{\mathrm{A}}^{+}{ }^{2} \mathrm{~A}_{1} \mathrm{H}_{2} \mathrm{O}^{+}$) after averaging over both photoemission direction and molecular orientation.

photon-ionization delays for the cases of $\mathrm{N}_{2} \mathrm{O}$ and $\mathrm{H}_{2} \mathrm{O}$ discussed in this article. The solid blue curve represents the one-photon-ionization delay of $\mathrm{N}_{2} \mathrm{O}$ following complete angular averaging. It displays a pronounced local maximum at a photon energy of $33 \mathrm{eV}$, i.e. again in the region of the shape resonance. The angle-and-orientation-averaged one-photonionization delays are however much smaller in magnitude than their only-angle-averaged counterpart in Fig. 7a. The effect of orientational averaging on one-photon-ionization delays in this case therefore mainly consists in reducing their magnitude. In contrast to this, orientational averaging has a much more dramatic effect on the two-photon-ionization delays. Comparing the red curve in Fig. 8a with the red curves in Fig. 7b, we find that the maximum in the orientation-averaged two-photon-ionization delays is shifted downwards by $\sim 7 \mathrm{eV}$ to a photon energy of $\sim 26 \mathrm{eV}$.

The seemingly surprising difference in the effects of orientational averaging on the oneand two-photon-ionization delays can be understood by inspection of Eqs. (6) and (19). Whereas the orientation dependence of the one-photon-ionization delays arises from the presence of a single Wigner rotation-matrix element, the orientation dependence of the twophoton matrix element involves a sum over products of two Wigner rotation-matrix elements. This more complex dependence enables much richer interference phenomena to take place in the coherent-averaging process. In the present case, we find that the orientational average 
can shift the position of the maxima in the two-photon-ionization delays by a substantial amount. The generality of this result will be investigated in future work.

In the case of the water molecule, illustrated in Fig. 8b, the differences between angleaveraged one- and two-photon delays are much less pronounced, which is most likely a consequence of the lack of structure in the photoionization continuum of $\mathrm{H}_{2} \mathrm{O}$ considered here. Both the one- and the two-photon-ionization delays monotonically decrease as a function of energy but the two-photon delays are slightly lower in magnitude than for the one-photon case. Finally, we point out that the theoretical results described in this article are supported by the experimental results reported in Ref. [29].

\section{CONCLUSIONS}

In this article we have developed a theory for calculating one- and two-photon ionization delays of arbitrary molecules using ab-initio quantum scattering calculations. Starting with an expression connecting the single-photon-ionization matrix elements and the concept of "time delay" as employed in the context of the analysis of scattering phenomena, the time delays encountered in molecular photoionization phenomena were found to be subject to pronounced variations as a function of energy, photoemission direction and target orientation. The highly anisotropic nature of the scattering potential experienced by the outgoing wavepacket gives rise to pronounced angular dependences of the photoionization time delay as revealed by both emission- and orientation-direction-resolved angular maps. By comparing the location of these features with results of energy-resolved measurements, in particular photoelectron spectroscopy, and results from molecular photoionization calculations, we were able to relate the angular structures to the presence of shape resonances in the continuum. This fact is clarified by comparing the one-photon-ionization delays in the two molecules $\mathrm{N}_{2} \mathrm{O}$ and $\mathrm{H}_{2} \mathrm{O}$ for photoionization initiating from the electronic ground states of the neutral species and terminating in the first excited electronic states of the cation. We find that the energy positions associated with the presence of shape resonances in the $7 \sigma \rightarrow k \sigma$ and $7 \sigma \rightarrow k \pi$-channels lead to rapid variations of the time delay as a function of angle in the case of $\mathrm{N}_{2} \mathrm{O}$, a situation that contrasts with the rather monotonic angular dependence of the time delays in the water molecule. These features were found to be very sensitive to averaging effects, both with respect to the photoemission and target-orientation directions. 
We then provided a formalism for calculating the two-photon matrix elements that bear relevance in interferometric measurements where the delays associated with the XUV-mediated photoionization step are entangled with the contributions of IR-induced transitions coupling different continua. Photoemission- and orientation-direction averaging can have significant effects on the observed total delays, leading even to an energy shift of the local maximum of the delay, associated with the position of a shape resonance, as demonstrated in the case of $\mathrm{N}_{2} \mathrm{O}$. In principle, the formalism presented in this article can easily be applied to other molecular targets, including more complex systems such as large polyatomic molecules or clusters, provided that the scattering matrix elements as defined by eq. (1) are available. Our work thus establishes the foundation for the calculation and theoretical analysis of the photoionization of molecules on attosecond time scales. Interesting applications and refinements of this work will include the analysis of electron correlation phenomena in molecular photoionization.

\section{ACKNOWLEDGMENTS}

We gratefully acknowledge funding from an ERC Starting Grant (contract 307270ATTOSCOPE) and the NCCR-MUST, a funding instrument of the Swiss National Science Foundation.

[1] A. L. Cavalieri, N. Muller, T. Uphues, V. S. Yakovlev, A. Baltuska, B. Horvath, B. Schmidt, L. Blumel, R. Holzwarth, S. Hendel, M. Drescher, U. Kleineberg, P. M. Echenique, R. Kienberger, F. Krausz, and U. Heinzmann, Nature 449, 1029 (2007).

[2] R. Locher, L. Castiglioni, M. Lucchini, M. Greif, L. Gallmann, J. Osterwalder, M. Hengsberger, and U. Keller, Optica, Optica 2, 405 (2015).

[3] Z. Tao, C. Chen, T. Szilvási, M. Keller, M. Mavrikakis, H. Kapteyn, and M. Murnane, Science 353, 62 (2016), http://science.sciencemag.org/content/353/6294/62.full.pdf.

[4] M. Schultze, M. Fiess, N. Karpowicz, J. Gagnon, M. Korbman, M. Hofstetter, S. Neppl, A. L. Cavalieri, Y. Komninos, T. Mercouris, C. A. Nicolaides, R. Pazourek, S. Nägele, J. Feist, J. Burgdörfer, A. M. Azzeer, R. Ernstorfer, R. Kienberger, U. Kleineberg, E. Goulielmakis, 
F. Krausz, and V. S. Yakovlev, Science 328, 1658 (2010).

[5] K. Klünder, J. M. Dahlström, M. Gisselbrecht, T. Fordell, M. Swoboda, D. Guénot, P. Johnsson, J. Caillat, J. Mauritsson, A. Maquet, R. Taïeb, R.ieb, and A. L'Huillier, Phys. Rev. Lett. 106, $143002(2011)$.

[6] D. Guénot, K. Klünder, C. L. Arnold, D. Kroon, J. M. Dahlström, M. Miranda, T. Fordell, M. Gisselbrecht, P. Johnsson, J. Mauritsson, E. Lindroth, A. Maquet, R. Taïeb, A. L'Huillier, and A. S. Kheifets, Phys. Rev. A 85, 053424 (2012).

[7] D. Gunot, D. Kroon, E. Balogh, E. W. Larsen, M. Kotur, M. Miranda, T. Fordell, P. Johnsson, J. Mauritsson, M. Gisselbrecht, K. Varj, C. L. Arnold, T. Carette, A. S. Kheifets, E. Lindroth, A. LHuillier, and J. M. Dahlstrm, Journal of Physics B: Atomic, Molecular and Optical Physics 47, 245602 (2014).

[8] E. P. Mansson, D. Guenot, C. L. Arnold, D. Kroon, S. Kasper, J. M. Dahlstrom, E. Lindroth, A. S. Kheifets, A. LH́uillier, S. L. Sorensen, and M. Gisselbrecht, Nat Phys 10, 207 (2014).

[9] C. Palatchi, J. M. Dahlstrm, A. S. Kheifets, I. A. Ivanov, D. M. Canaday, P. Agostini, and L. F. DiMauro, Journal of Physics B: Atomic, Molecular and Optical Physics 47, 245003 (2014).

[10] M. Sabbar, S. Heuser, R. Boge, M. Lucchini, T. Carette, E. Lindroth, L. Gallmann, C. Cirelli, and U. Keller, Phys. Rev. Lett. 115, 133001 (2015).

[11] M. Kotur, D. Guenot, A. Jimenez-Galan, D. Kroon, E. W. Larsen, M. Louisy, S. Bengtsson, M. Miranda, J. Mauritsson, C. L. Arnold, S. E. Canton, M. Gisselbrecht, T. Carette, J. M. Dahlstrom, E. Lindroth, A. Maquet, L. Argenti, F. Martin, and A. L'Huillier, Nat Commun 7 (2016).

[12] A. S. Kheifets and I. A. Ivanov, Phys. Rev. Lett. 105, 233002 (2010).

[13] J. M. Dahlström, A. L'Huillier, and A. Maquet, Journal of Physics B: Atomic, Molecular and Optical Physics 45, 183001 (2012).

[14] J. Dahlström, D. Guénot, K. Klünder, M. Gisselbrecht, J. Mauritsson, A. L'Huillier, A. Maquet, and R. Taieb, Attosecond spectroscopy, Chemical Physics 414, 53 (2013).

[15] A. S. Kheifets, Phys. Rev. A 87, 063404 (2013).

[16] S. Neppl, R. Ernstorfer, E. M. Bothschafter, A. L. Cavalieri, D. Menzel, J. V. Barth, F. Krausz, R. Kienberger, and P. Feulner, Phys. Rev. Lett. 109, 087401 (2012). 
[17] S. Neppl, R. Ernstorfer, A. L. Cavalieri, C. Lemell, G. Wachter, E. Magerl, E. M. Bothschafter, M. Jobst, M. Hofstetter, U. Kleineberg, J. V. Barth, D. Menzel, J. Burgdorfer, P. Feulner, F. Krausz, and R. Kienberger, Nature 517, 342 (2015).

[18] R. Pazourek, S. Nagele, and J. Burgdörfer, Rev. Mod. Phys. 87, 765 (2015).

[19] S. Kawai and A. D. Bandrauk, Phys. Rev. A 75, 063402 (2007).

[20] J. C. Baggesen and L. B. Madsen, Phys. Rev. A 83, 021403 (2011).

[21] I. A. Ivanov, A. S. Kheifets, and V. V. Serov, Phys. Rev. A 86, 063422 (2012).

[22] X.-F. Hou, L.-Y. Peng, Q.-C. Ning, and Q. Gong, Journal of Physics B: Atomic, Molecular and Optical Physics 45, 074019 (2012).

[23] V. V. Serov, V. L. Derbov, and T. A. Sergeeva, Phys. Rev. A 87, 063414 (2013).

[24] Q.-C. Ning, L.-Y. Peng, S.-N. Song, W.-C. Jiang, S. Nagele, R. Pazourek, J. Burgdörfer, and Q. Gong, Phys. Rev. A 90, 013423 (2014).

[25] J. Caillat, A. Maquet, S. Haessler, B. Fabre, T. Ruchon, P. Salières, Y. Mairesse, and R. Taïeb, Phys Rev Lett 106, 093002 (2011).

[26] A. Chacon, M. Lein, and C. Ruiz, Phys. Rev. A 89, 053427 (2014).

[27] P. Hockett, E. Frumker, D. M. Villeneuve, and P. B. Corkum, Journal of Physics B: Atomic, Molecular and Optical Physics 49, 095602 (2016).

[28] H. J. Wörner, in Proceedings of the $3^{\text {rd }}$ international conference on "Correlation Effects in Radiation Fields" (Sept. 13-18, 2015).

[29] M. Huppert, I. Jordan, D. Baykusheva, A. von Conta, and H. J. Wörner, to be published (2016).

[30] J. Cooper and R. N. Zare, The Journal of Chemical Physics 48, 942 (1968).

[31] E. P. Wigner, Phys. Rev. 98, 145 (1955).

[32] L. Eisenbud, Formal properties of nuclear collisions, Ph.D. thesis (Princeton University, 1948).

[33] F. T. Smith, Phys. Rev. 118, 349 (1960).

[34] A. P. Martin, Acta Physica Austriaca 23, 157 (1981).

[35] D. Boll, F. Gesztesy, and H. Grosse, Journal of Mathematical Physics 24, 1529 (1983).

[36] M. L. Goldberger and K. M. Watson, Phys. Rev. 127, 2284 (1962).

[37] P. M. Paul, E. S. Toma, P. Breger, G. Mullot, F. Augé, P. Balcou, H. G. Muller, and P. Agostini, Science 292, 1689 (2001). 
[38] F. A. Gianturco, R. R. Lucchese, and N. Sanna, The Journal of Chemical Physics 100, 6464 (1994).

[39] A. P. P. Natalense and R. R. Lucchese, The Journal of Chemical Physics 111, 5344 (1999).

[40] R. R. Lucchese, K. Takatsuka, and V. McKoy, Physics Reports 131, 147 (1986).

[41] R. R. Lucchese and D. Dowek, "Angular distributions in molecular photoionization," in Attosecond and XUV Physics (Wiley-VCH Verlag GmbH \& Co. KGaA, 2014) pp. 293-320.

[42] H. Park and R. N. Zare, The Journal of Chemical Physics 104, 4554 (1996).

[43] E. P. Wigner, Phys Rev A 98, 145 (1955).

[44] F. T. Smith, Phys. Rev. 118, 349 (1960).

[45] H. M. Nussenzveig, Phys. Rev. D 6, 1534 (1972).

[46] In the case of collision scattering, the above equation needs to be modified to exclude the contribution at $\hat{k} \equiv\left(\theta_{k}, \phi_{k}\right)=(0,0)$ that gives rise to an interference with the unscattered wave - a problem that does not arise in the context of photoionization.

[47] R. R. Lucchese, G. Raseev, and V. McKoy, Phys. Rev. A 25, 2572 (1982).

[48] H. Stapelfeldt and T. Seideman, Rev. Mod. Phys. 75, 543 (2003).

[49] P. M. Kraus, A. Rupenyan, and H. J. Wörner, Phys. Rev. Lett. 109, 233903 (2012).

[50] P. M. Kraus, D. Baykusheva, and H. J. Wörner, Phys. Rev. Lett. 113, 023001 (2014).

[51] P. M. Kraus, D. Baykusheva, and H. J. Wörner, J. Phys. B 47, 124030 (2014).

[52] U. Fano, Phys. Rev. A 32, 617 (1985).

[53] C. Brion and K. Tan, Chemical Physics 34, 141 (1978).

[54] C. M. Truesdale, S. Southworth, P. H. Kobrin, D. W. Lindle, and D. A. Shirley, The Journal of Chemical Physics 78, 7117 (1983).

[55] T. A. Carlson, P. R. Keller, J. W. Taylor, T. Whitley, and F. A. Grimm, The Journal of Chemical Physics 79, 97 (1983).

[56] E. Poliakoff, M.-H. Ho, M. White, and G. Leroi, Chemical Physics Letters 130, 91 (1986).

[57] M. Braunstein and V. McKoy, The Journal of Chemical Physics 87, 224 (1987).

[58] T. A. Ferrett, A. C. Parr, S. H. Southworth, J. E. Hardis, and J. L. Dehmer, The Journal of Chemical Physics 90, 1551 (1989).

[59] L. A. Kelly, L. M. Duffy, B. Space, E. D. Poliakoff, P. Roy, S. H. Southworth, and M. G. White, The Journal of Chemical Physics 90, 1544 (1989). 
[60] R. T. Wiedmann, E. R. Grant, R. G. Tonkyn, and M. G. White, The Journal of Chemical Physics 95, 746 (1991).

[61] M. Braunstein and V. McKoy, The Journal of Chemical Physics 87, 224 (1987). 